\title{
Pareto Optimization of Power System Reconstruction Using NSGA-II algorithm
}

\author{
Hongtao WANG \\ School of Electrical Engineering \\ Shandong University \\ Jinan, China \\ whtwhm@sdu.edu.cn
}

\author{
Chengming HE \\ School of Electrical Engineering \\ Shandong University \\ Jinan, China \\ sduhcm@yahoo.cn
}

\author{
Yutian LIU \\ School of Electrical Engineering \\ Shandong University \\ Jinan, China \\ liuyt@sdu.edu.cn
}

\begin{abstract}
After blackout, power system reconstruction is a multiobjective optimization problem, especially for parallel restoration through power system partitioning. It includes optimal systempartitioning strategy, units optimal starting-up sequence, time requirements for system reconstruction. A constrained multiobjective optimization model is proposed for power system reconstruction. Fast and elitist non-dominated sorting genetic algorithm (NSGA-II) is applied in order to avoid artificially balanced solutions. Priority-based genetic encoding and decoding are designed for NSGA-II. With non-dominated sorting and crowding distance calculation the means of dummy fitness are used to find Pareto optimal solutions. An elitist checking strategy is adopted to check Pareto optimal solutions. Simulation results on IEEE 30-bus system show that the proposed algorithms are able to find better spread of solutions, better convergence near the Pareto-optimal front and lower computational complexity compared to other power system reconstruction methods based on genetic algorithms. The effectiveness of the proposed algorithms is further validated by the numerical results on the power system of Shandong province, China.
\end{abstract}

Keywords-power system restoration; genetic algorithm; multi objective optimization; power system reconstruction

\section{INTRODUCTION}

Power system restoration is complex problem for decision making. It is always divided into three phases included blackstart, system reconstruction and load recovery [1]. Compared with the research of service restoration in distribution system for decades, the study of power system reconstruction is not yet immature. The objectives become diversified and synthetically. The objectives of power system reconstruction include the maximizing restoration generator capacity, minimizing time for system reconstruction and maximizing the load recovery [2-8].

In those papers, the multi-objective reconstruction problem is always handled as a single objective problem with constraint method [2] or weighted method [3-8]. The constraint method is essentially single-objective programming. And for weighted method, the results are seriously influenced by weighted factor. But the presence of multiple objectives in the problem, in principle, gives rise to a set of optimal solutions, instead of a single optimal solution. In the absence of any further information, one of these Pareto-optimal solutions cannot be said to be better than the other. This demands a user to find as many Pareto-optimal solutions as possible. Classical optimization methods suggest converting the multi-objective optimization problem to a single-objective optimization problem by emphasizing one particular Pareto-optimal solution at a time. When such a method is to be used for finding multiple solutions, it has to be applied many times, hopefully finding a different solution at each simulation run.

Fast and Elitist Non-dominated Sorting in Genetic Algorithms (NSGA-II) is a new multi-objective Genetic Algorithms [9]. The individual fitness degrees embody in the rank of non-dominated sorting and crowding distances. It avoids objective bias character due to artificial factor weights and acquires the solution set with optimum in every objective as much as possible. It provides an effective method for balancing among various objectives [10].

NSGA-II is applied in multi-objective system reconstruction. Simulation results on IEEE 30-bus system show that the proposed algorithms are able to find better spread of solutions, better convergence and lower computational complexity compared to other power system reconstruction methods based on genetic algorithms. The effectiveness of the proposed algorithms is further validated by the numerical results on the power system of Shandong province, China.

\section{Multi-ObJective System Reconstruction Model}

\section{A. Multi-Objective Function}

Three optimal objectives are considered in the same time:1) Restoration maximize generating capacity in limited time;2) Optimization of parallel restoration through power system sub area; 3) Minimum time for system skeleton reconstruction including all destination nodes.

$$
\left\{\begin{array}{l}
\min \left(f_{1}, f_{2}, f_{3}\right) \\
f_{1}=-\sum_{s=1}^{k} P_{s \max } \\
f_{2}=T_{\max } \\
f_{3}=\sum_{s=1}^{k} T_{s}
\end{array}\right.
$$

This work was supported by Chinese National Natural Science Foundation of China (No. 50877044), Young and Middle-aged Scientist Reward Foundation of Shandong Province in China (2008BS01006). 
Where: $f_{1}$ represents the sum of generation capacity already been started in the critical start time limit. $k$ is the number of black start sources(or subareas). $P_{\text {smax }}$ is the sum of generation capacity already been started in the $s$ th subarea. $f_{2}$ corresponds to $T_{\max }=\max \left\{T_{1}, T_{2}, \cdots, T_{\mathrm{k}}\right\}$ which is maximum restoration time among different system partition. $f_{3}$ corresponds to the sum time for system skeleton reconstruction including all destination nodes. $T_{\mathrm{s}}$ is the system skeleton reconstruction time for the $s$ th system partition.

\section{B. Constraint}

For partition $s=1,2, \cdots, k$, there are following constraints:

1) Time Constraint

$$
0<T_{r j}<T_{m c j}, \quad j=1,2, \ldots, N_{s g}
$$

Where: $T_{\mathrm{rj}}$ is time which non black-start generator waits for ac power from black-start sources. $T_{\mathrm{mcj}}$ is the time limited for generator hot start constraint; $N_{\mathrm{sg}}$ is the sum of generators in $s$ th subarea.

\section{2) Power Flow and Node Voltage Constraint}

$$
\begin{array}{ll}
P_{G i}^{\min } \leq P_{G i} \leq P_{G i}^{\max }, & i=1,2, \ldots, N_{s g} \\
Q_{G i}^{\min } \leq Q_{G i} \leq Q_{G i}^{\max }, & i=1,2, \ldots, N_{s g} \\
P_{i} \leq P_{i \max } & i=1,2, \ldots, b \\
U_{i}^{\min } \leq U_{i} \leq U_{i}^{\max }, & i=1,2, \ldots, N_{s n}
\end{array}
$$

Where: $N_{s n}$ is the sum of nodes in $s$ th subarea; $P_{i}$ is active power flow through branch $i$; $P_{\text {imax }}$ is the maximum allowable active power flow through branch $i ; b$ is the number of all branches; $U_{i}$ is the node voltage;

\section{3) Generator Excitation Constraint;}

$$
K S_{e}>Q_{\Sigma c}
$$

Where: $S_{\mathrm{e}}$ is the capacity of black start generator; $K$ is the short circuit ratio (SCR) of black start generator; $Q_{\Sigma \mathrm{c}}$ is reactive charging power for transmission lines.

\section{PARETO OPTIMIZATION SOLVED BY NSGA-II}

\section{A. Pareto Optimal}

There are several objectives for multi-objective restoration optimal problem which has same decision variables. Influenced by mutual restrictive relationship between each decision variables, it can't reach optimal in any objective direction with the same decision variables. Then Pareto optimal solution is introduced for the multi-objective problem.

For multi-objective problems, if the objective function of $x^{(1)}$ satisfy formulation (5) and (6), then solution $x^{(1)}$ is called dominating solution $x^{(2)}$.

$$
\begin{gathered}
f_{j}\left(x^{(1)}\right) \leq f_{j}\left(x^{(2)}\right), \quad \forall \mathrm{j}=1,2, \ldots, M \\
f_{j}\left(x^{(1)}\right)<f_{j}\left(x^{(2)}\right), \quad \exists \mathrm{j} \in\{1,2, \ldots, M\}
\end{gathered}
$$

Where: $M$ is objective function.

Solution $x^{(1)}$ is also called non-dominated solution. Solution $x^{(2)}$ is called dominated solution. If there is not any solution dominating solution $x^{*}, x^{*}$ is called Pareto optimal solution. Pareto optimal solution set denoted by $X^{*}$ is the set with all Pareto optimal solutions. For objective functions $f(x)$ and Pareto optimal solution set $X^{*}$, Pareto front $V$ is defined by $V=\left\{u=f(x) \mid x \in X^{*}\right\}$.

\section{B. NSGA-II Algorithm}

There are three performance evaluation indicators for Pareto optimal algorithm: (a) The solution should close to Pareto optimal solution; (b) Solution set should uniformly distribute; (c) Pareto solution acquired should not be lost. Accordingly there are three key technologies including 'fast non-nominate sorting', 'crowding distance' and 'elitist strategy' in NSGA-II outperforms the traditional multiobjective algorithms. Based on the principles of NSGA-II, three kinds of operators are designed for multi-objective system reconstruction:

\section{1) Operator of Fast Non-Dominate Sorting}

This operator which is based on the rank of non-dominate which leads the solution set close to Pareto optimal solution set. It is a cycling procedure of rating: (a) Perform nondominated sorting on population $R_{i}$. The solutions in $R_{i}$, which do not dominate each other but dominate all the other solutions of $R_{i}$, are kept in the first front or best front (called set $F_{1}$ ). (b) Among the solutions not in $F=F_{1}$, the solutions which do not dominate each other but dominate all the other solutions, are kept in the second front (called set $F_{2}$ ). (c) This process is repeated until there is no solution in $R_{i}$ without having its own front. Subsequently, these generated fronts are assigned their corresponding ranks. Thus, $F_{1}$ is assigned rank $1 ; F_{2}$ is assigned rank 2 and so on.

\section{2) Operator of Crowding Distance}

To choose solutions from the same non-dominated front, solutions are further sorted according to their crowding distances. The crowding distance computation requires sorting the population according to each objective function value in ascending order of magnitude. The crowding distance of the $i$ th solution in its front is the distance between $i+1$ th and $i-1$ th solutions: (a) for each objective function, the boundary solutions (solutions with smallest and largest function values) are assigned an infinite distance value; (b) all other intermediate solutions are assigned a distance value equal to the absolute normalized difference in the function values of two adjacent solutions. This calculation is continued with other objective functions; (c) the overall crowding distance value is calculated as the sum of individual distance values corresponding to each objective. Each objective function is normalized before calculating the crowding distance. The crowded-comparison operator, which prefers the solution that 
has larger crowding distance, guides the selection process at the various stages of the algorithm toward a uniformly spreadout Pareto-optimal front.

\section{3) An Elitist Preserving Approach}

An elitist-preserving approach plays a role to avoid missing Pareto-optimal solution from parent population. To create the next generation $P_{i+1}$, the following procedure is adopted: (a) Solutions which is marked as unfeasible solution through verification procedure are discarded firstly; (b) $P_{i+1}$ is filled up with the parent and offspring population according to the non-dominate sorting rank from low to high until the total number of solutions $n$ is greater than population scale $N$. (c) To make the size of $P_{i+1}$ exactly equal to $N,(n-N)$ solutions from the last included non-dominated front are discarded from $P_{i+1}$. To choose the solutions to be discarded, initially the solutions of the last included non-dominated front are sorted according to their crowding distances and subsequently, the solutions having least $(n-N)$ crowding distances are discarded from $P_{i+1}$.

\section{GRID RECONSTRUCTION BASED ON NSGA-II}

\section{A. Gene Coding}

\section{1) Chromosome Structural Design}

For each destination node to be restored, because it is necessary to determine the partition it is located, but also the restoration sequence, so the chromosome structure of this paper is as follows: Assumption that the system includes $e$ destination nodes to be restored and $k$ black-start sources, then solutions can be expressed as the chromosome $\left(i_{1}, i_{2}, \ldots, i_{\mathrm{e}}\right.$ । $\left.A_{1}, A_{2}, \ldots, A_{h}\right)$. The previous $e$-bit segment represents the sorting operation codes. The last $h$-bit segment represents partition operation codes. The segment $\left\{A_{1}, A_{2}, \ldots, A_{h}\right\}$, which contains $k$ different elements indicated $k$-partitions, it represents the corresponding node is assigned to a certain partition. Usually $h$ $\leqslant e$, since the partition property for some node there is only one choice, they will not be allocated partition codes. The way of gene decoding is based on priority. The smaller the code value in the sorting segment has the higher priority, corresponding to the order of the node to restore the more priority. For the partition segment, if code value less than or equal 0.5 in the condition of two partitions system, then corresponding node is divided to $A$ zone, otherwise $B$ zone. An example is shown in Fig 1.

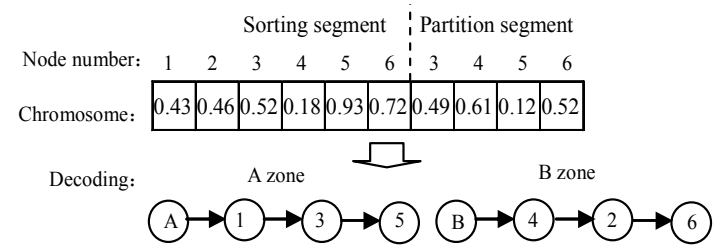

Figure 1. Sketch map of priority-based genetic encoding and decoding.

\section{2) System Reconstruction Based on Chromosome}

Chromosome code provides the node information of sequence for power system reconstruction. Based on the rule of gene encoding and decoding, it can be reduced to a reasonable reconstruction of the grid. First, chromosome is decoded, and then the restoration sequence of destination nodes and system division are fixed; second, find the shortest recovery path for the destination nodes starting from the established black start source, and record the recovery time of each node; finally, the objective function can be calculated according to the reconstruction procedures. Dijkstra algorithm is used for calculating shortest path. The edge weights are measured by time consuming with line operation.

\section{B. Algorithm Based on NSGA-II}

Based on the three operator of NSGA-II, the multiobjective grid construction algorithms consist of the following modules:

\section{1) Population Initialization}

The incipient population is generated base on the designed genetic code, each individual represents one restoration scheme, then generate the relevant restoration scheme with the grid reconstruction algorithm, at last, calculate the adaptive value of each objective.

\section{2) Binary Tournament Selection}

Two random parent individuals are compared in the binary tournament selection, individual $j$ will be eliminated while $i_{\text {rank }}<j_{\text {rank }}$ or $L[i]_{\mathrm{d}}>L[j]_{\mathrm{d}}$ if $i_{\text {rank }}=j_{\text {rank. }}$. In other words, the one with the smaller non-dominated sorting will be maintained if nondominated sorting of two individuals are different; if the two have the same non-dominated sorting, select the one with a larger crowding distance. In this way, the result can be welldistributed and the diversity of the population can be maintained.

\section{3) Crossover and Mutation}

In order to obtain good performance of global optimization, SBX (Simulated Binary Crossover) operator and random mutation operator are used in the algorithm. These operators act on the population selected by binary tournament selection to generate the offspring $Q_{i}$.

\section{4) Elite Verification Strategy}

There are three functions used to check the elitist individual: scheme decoding, elite verification and scheme adjustment. Scheme decoding is to decode the new parent chromosome selected by elite strategy, and then reconstruct the corresponding restoration sequence. It's necessary to check whether the restoration sequence is feasible with the constraint of power flow, node voltage and generator excitation. All of the feasible one will be marked as feasible and others are marked as unfeasible, during this process the marked chromosomes can be overleaped. The function of scheme adjustment is to adjust the scheme which exceeds the power flow limit. The output of generator and load to pickup are adjusted with sensitivity analysis method. If the regulating quantity within the limit, the corresponding scheme is feasible, otherwise it will be marked as unfeasible. During the next round of evolution, the scheme marked as unfeasible will be eliminated without check. The mark of new chromosome will be eliminated after crossover and mutation. Selected by elite strategy, the one with feasible mark will be maintained without check.

To sum up, flow chart of multi-objective power system reconstruction based on NSGA-II is given in Fig. 2 . 


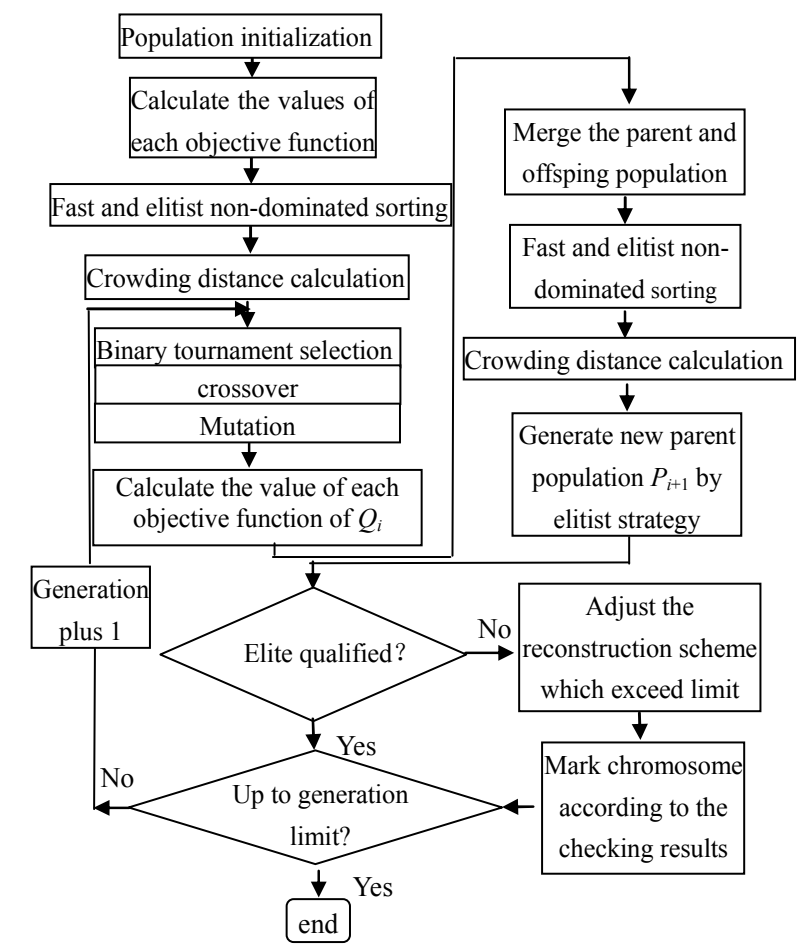

Figure 2. Flow chart of multi-objective power system reconstruction based on NSGA-II

\section{EXAMPLE}

Through programming the multi-objective power system reconstruction based on NSGA-II, the effectiveness of the algorithm is validated by the numerical results on IEEE 30-bus system and the practical Shandong power system.

\section{A. IEEE 30-Bus System}

Under the assumption that there are two partitions in IEEE 30-bus system, bus 1 and 2 represent the black start node of partition $A$ and partition $B$. SCR $K$ is given a value of 0.827 . The value of $K S_{e}$ is $0.827 * 80=66.16$ (MVar). When the black start node has started, the following destination generator nodes are $\{13,22,23,27\}$ and the load nodes are $\{7,12,17,19,21,30\}$. In Fig. 3, the weights marked on the edge represent restoration time (hypothetical value, min), the hot start time of generator node 27 is $10 \mathrm{~min}$, and others are $30 \mathrm{~min}$ [6].

According to the sum of nodes, there are 10 sorting operation codes and 7 partition operation codes. For node 7, 12 and 13 , there is only one choice, so they don't need partition operation codes. Table 1 gives the statistical results of 400 simulation tests, in which $\mathrm{P}\left(V^{\prime}=\mathrm{U}\right)$ is probability of the Paretooptimal solution $V$ of each simulation equals to $U$ which contain all the $V^{\prime}$. With mutation rate 0.2 , it is always reaching the Pareto front within 40 generation and has stable convergence with the probability above $90 \%$.

The Pareto-optimal solution and the corresponding Pareto front obtained by elitist selection are shown in table 2. In Fig. 3, the result of network reconfiguration in the scheme 2 is shown with solid line and the bold line represents the boundary between two sub-areas. Differing from the traditional genetic algorithm which will check power flow of each restoration sequence, the elite verification strategy reduces the calculating amount apparently.

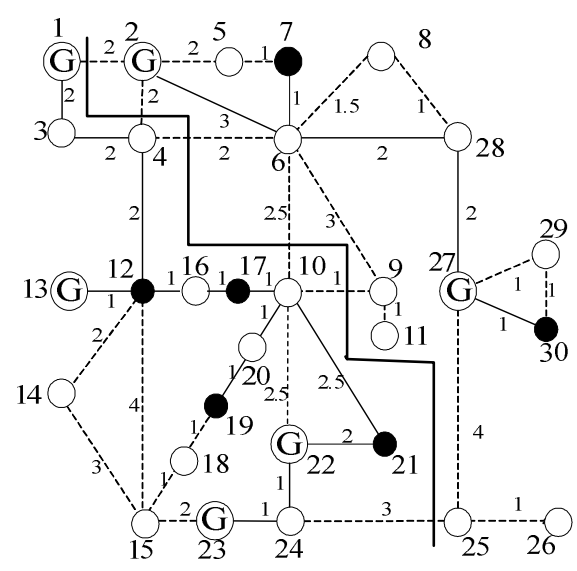

Figure 3. Partitioning results of IEEE 30-bus system

Under the same calculating condition, using genetic algorithm we can get scheme 1 [6]. Comparing the objective function value of the two schemes, we know that the three objective functions of scheme 1 and scheme 2 don't dominate each other. They all belong to the Pareto-optimal solution. Plan 1 is better on the objective of the maximum sub-area recovery time and plan 2 is better on the objective of the average recovery time. Comparing with the genetic algorithm, the solution set obtained by NSGA-II algorithm distribute better in each objective space.

TABLE I. STATISTICAL COMPARISON IN SIMULATIONS FOR THE IEEE 30-BUS SYSTEM

\begin{tabular}{|c|c|c|c|c|c|}
\hline Serial no. & Population & $\begin{array}{c}\text { Mutation } \\
\text { Rate }\end{array}$ & $\begin{array}{c}\text { Experiment } \\
\text { Times }\end{array}$ & $\begin{array}{c}\text { Average } \\
\text { Convergent } \\
\text { Generation }\end{array}$ & $\mathbf{P}\left(\mathbf{V}^{\prime}=\mathbf{U}\right)$ \\
\hline 1 & 100 & 0.2 & 100 & 32 & $92 \%$ \\
\hline 2 & 50 & 0.2 & 100 & 24 & $90 \%$ \\
\hline 3 & 100 & 0.1 & 100 & 13 & $86 \%$ \\
\hline 4 & 50 & 0.1 & 100 & 16 & $83 \%$ \\
\hline
\end{tabular}

TABLE II. SCHEMES OF IEEE30 SYSTEM RECONSTRUCTION

\begin{tabular}{|c|c|c|c|c|c|}
\hline $\begin{array}{c}\text { Scheme } \\
\text { no. }\end{array}$ & $\begin{array}{c}\text { Restoration } \\
\text { Sequence in } \\
\text { Partition 1 }\end{array}$ & $\begin{array}{c}\text { Restoration } \\
\text { Sequence in } \\
\text { Partition 2 }\end{array}$ & $\begin{array}{c}\boldsymbol{f}_{\mathbf{1}} \\
(\mathbf{M W})\end{array}$ & $\begin{array}{c}\boldsymbol{f}_{\mathbf{2}} \\
(\mathbf{m i n})\end{array}$ & $\begin{array}{c}\boldsymbol{f}_{\mathbf{3}} \\
(\mathbf{m i n})\end{array}$ \\
\hline 1 & $12,13,23,22,21$ & $27,30,19,17,7$ & 175 & 17 & 15.75 \\
\hline 2 & $\begin{array}{c}12,13,17,21,22, \\
23,19\end{array}$ & $27,7,30$ & 175 & 18.5 & 13.75 \\
\hline
\end{tabular}

\section{B. Shandong Power System}

To further authentication algorithm in large-scale systems, Shandong power grid with 172 stations (220kV and above), 396 transmission lines, 15 power plants is used as test system. There are two black start sources. One is Taian pumped storage power station, the other is external power supporting from the boundary Binzhou Station. As shown in Fig. 4, the destination load node is marked with solid black node, and the unit nodes to restore are marked with dot. Through calculation and verification, two solutions of Pareto optimal are obtained. Significant differences between them are the division of the 
system. One is east-west division; the other is south-north division. Fig. 4 shows the solution of east-west division which is dominant in $f_{1}$. The solution of south-north division is dominant in total restoration time. On this basis, the dispatcher is convenient to choose according to actual needs. Table 3 shows the statistical results of 600 times simulations in which fast convergence and better stability with the setting of 100 populations, 0.2 mutation rate.

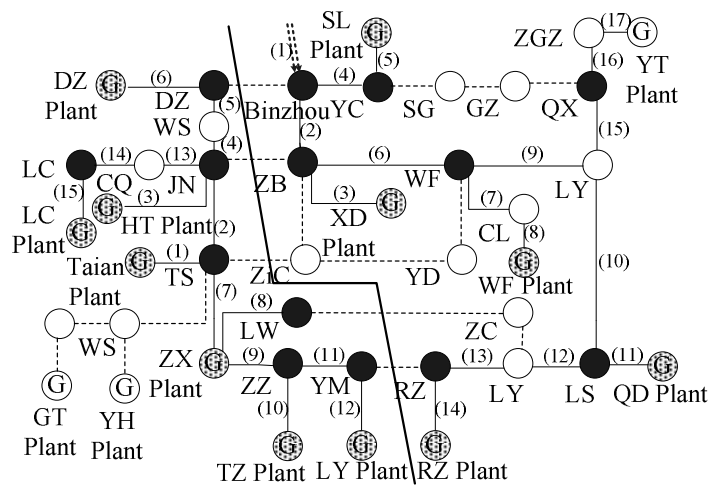

note: the number in the parentheses is the order of restoration sequence

Figure 4. Reconstruction with partitioning for the Shandong power system

TABLE III. STATISTICAL COMPARISON IN SIMULATIONS FOR THE SHANDONG SYSTEM

\begin{tabular}{|c|c|c|c|c|c|}
\hline $\begin{array}{c}\text { Serial } \\
\text { no. }\end{array}$ & Population & $\begin{array}{c}\text { Mutation } \\
\text { Rate }\end{array}$ & $\begin{array}{l}\text { Experiment } \\
\text { Times }\end{array}$ & $\begin{array}{c}\text { Average } \\
\text { Convergent } \\
\text { Generation }\end{array}$ & $\mathbf{P}\left(\mathbf{V}^{\prime}=\mathbf{U}\right)$ \\
\hline 1 & 100 & 0.2 & 100 & 61 & $91 \%$ \\
\hline 2 & 50 & 0.2 & 100 & 79 & $90 \%$ \\
\hline 3 & 100 & 0.1 & 100 & 27 & $86 \%$ \\
\hline 4 & 50 & 0.1 & 100 & 29 & $83 \%$ \\
\hline 5 & 100 & 0.5 & 100 & $>200$ & $90 \%$ \\
\hline 6 & 50 & 0.5 & 100 & $>200$ & $89 \%$ \\
\hline
\end{tabular}

\section{CONCLUSION}

A multi-objective optimization model is proposed for power system reconstruction after blackout. An algorithm based on NSGA-II has been suggested. The advantages of the proposed NSGA-II based technique are that it does not require any weighting factor and the strategy of elite verification apparently reduces the computation cost. The proposed technique has been found to be superior to the conventional GA based technique in the sense that it always attains the better restoration solution in lesser time compared to the conventional GA with IEEE 30-bus system. Also, it has been found that, the speed of convergence can be increased significantly. In practical large-scale power system tests, the Pareto optimization results offer more choices for dispatchers. In summary, it provides a new insight and tool for Pareto optimization of the multi-objective power system reconfiguration.

\section{REFERENCES}

[1] J. A. Jerry, "A framework for power system restoration following a major power failure," IEEE Transactions on Power Systems, vol. 10, no. 3, pp. 1480-1485, 1995.

[2] Y. H. Zhou and Y Min, "Optimal algorithm for system reconstruction," Proceedings of the CSEE, vol. 23, no. 4, pp. 67-70, 2003.

[3] Z. Y. Zhang, Y. P. Chen, and R. X. Yuan, "Fuzzy multiple attribute decision-making method for units' optimal starting-up sequence during the system reconstruction". Transactions of China Electrotechnical Society, vol. 22, no. 11, pp. 153-157, 2007

[4] H. H. Wang, Y. S. Xue, Z. Y. Dong , G. Ledwich, and Y. T. Liu, "Adaptive optimal restoration control for interconnected grids," Automation of Electric Power Systems, vol. 31, no. 22, pp. 1-5, 2007.

[5] Y. T. Liu and C. Y. Wang, "A group intelligent decision support system for power system skeleton restoration based on data warehouse," Automation of Electric Power Systems, vol. 33, no. 1, pp. 45-50, 2009.

[6] Z. Z. Liu and F. H. Wen, "A new optimization method for determining restoration paths based on weighted complex network model," Automation of Electric power systems, vol. 33, no. 6, pp. 11-15, 2009.

[7] X. G. Ping, H. Z. Hui, and H. P. Liang, "Optimization of parallel restoration through power system partitioning after blackout," Proceedings of the CSEE, vol. 29, no. 10, pp. 41-46, 2009.

[8] Q. Liu, L. S. Shi, Y. X. Ni, and Z. Y. Dong, "Intelligent optimization strategy of the power grid reconfiguration during power system restoration," Proceedings of the CSEE, vol. 29, no. 13, pp. 8-15, 2009.

[9] D. Kalyanmoyd, P. Amrit, A. Sameer, and T. Meyarivan, "A fast and elitist multi-objective genetic algorithm: NSGA-II," IEEE Transactions on Evolutionary Computation, vol. 6, no. 2, pp. 182-197, 2002.

[10] K. Yogendra, D. Biswarup, and S. Jaydev, "Service restoration in distribution system using non-dominated sorting genetic algorithm," Electric Power System Research, vol. 76, pp. 768-777, 2006. 